\title{
Antibacterial and catalytic properties of silver nanoparticles loaded zeolite: green method for synthesis of silver nanoparticles using lemon juice as reducing agent
}

\author{
J. Selvamuthumari ${ }^{1}$, S. Meenakshi ${ }^{2}$, M. Ganesan ${ }^{1}$, S. Nagaraj ${ }^{3}$, K. Pandian ${ }^{2, *}$ \\ ${ }^{1}$ Department of Chemistry, Thiagarajar College, Madurai-625009, India \\ ${ }^{2}$ Department of Inorganic Chemistry, University of Madras, Guindy Campus, Chennai-600025, India \\ ${ }^{3}$ CAS Botany, University of Madras, Guindy Campus, Chennai-600025, India \\ *jeevapandian@yahoo.co.uk
}

PACS 82.65.+r

DOI $10.17586 / 2220-8054-2016-7-4-768-773$

\begin{abstract}
Zeolite $\mathrm{Y}$ is a cage-like alumina silicate which is widely used as solid support to immobilize metal and metal sulfide nanoclusters. We have attempted to synthesis silver nanoparticle-loaded zeolite $\mathrm{Y}$ by an ion exchange method followed by a biogenic reduction method using lemon juice as a reducing agent. The antimicrobial activity of the silver ion, silver nanoparticles and silver chloride-modified zeolite was investigated against various Gram negative and Gram positive microorganisms. The silver nanoparticle-loaded zeolite was further functionalized with amoxicillin antibiotic which exhibited a strong antimicrobial action to kill drug resistant microorganisms. The catalytic behavior of silver nanoparticles was investigated to reduce 4-Nitrophenol in presence of $\mathrm{NaBH}_{4}$. The catalytic reaction is found to be pseudo-first order, resulting in a rate constant that was comparable with previously-reported results.
\end{abstract}

Keywords: zeolite-Y, silver nanoparticles, amoxicillin, catalytic reduction of 4-aminophenol, antibacterial studies.

Received: 3 May 2016

Revised: 25 June 2016

\section{Introduction}

Zeolites are an important group of crystalline aluminosilicates currently available for various fields of applications. These minerals are widely used as sorbents, ion exchangers, catalysts and biosensors [1-4]. The catalytic nature, reactivity and other properties of zeolite can be greatly improved by cation exchange methods [5]. These materials are negatively charged with a high density of active acid sites, high thermal stability, high size selectivity and unique porous properties, which impart their ability act as catalysts for various industrial catalytic studies at elevated temperature with wider $\mathrm{pH}$ ranges. Because of void space and swelling behavior which can be used in petrochemical cracking, ion-exchange, gas and solvent separation, and removal of pollutants [6]. Metal ion, charged species, redox and photoactive molecules can be immobilized within the pores of zeolite system and these composites can be used in sensors and catalytic applications [7-10].

Silver (Ag) is a metallic element that has been widely used in various excellent fields [11]. Silver ions $\left(\mathrm{Ag}^{+}\right)$and silver nanoparticles are effective in inhibiting bacterial growth and may damage the DNA of both Gram-positive and Gram-negative bacteria [12-15]. Silver modified zeolite has been used in various fields such as catalyst, biosensors, water purification, antifungal and antimicrobial activity $[16,17]$.

The aim of this study is to prepare AgNPs/Zeo-Y nanocomposites using lemon juice as reducing agent by green synthesis method and study of its applications in the catalytic reduction of 4-Nitrophenol and the antibacterial activity of the system.

\section{Experimental Section}

\subsection{Chemicals}

Zeolite-Y and silver nitrate were purchased from Sigma Aldrich, USA. 4-Nitrophenol and liquid ammonia $(30 \% \mathrm{v} / \mathrm{v})$ were received from Fisher Scientific Pvt. Ltd., India. Sodium borohydrate, sodium chloride and amoxicillin were received from Merck, India. Hydrochloric acid and sulphuric acid were purchased from SRL Pvt. Ltd., India. All reagents and chemicals were used received from commercial source with an analytical grade and without further purification. 


\subsection{Preparation of AgNPs/Zeo-Y}

To prepare AgNPs/Zeo-Y nanocomposite, $0.1 \mathrm{M} \mathrm{AgNO}_{3}$ was dissolved in $20 \mathrm{~mL}$ of distilled water and then $1 \%$ solution of $\mathrm{NH}_{4} \mathrm{OH}$ was added dropwise to the aq. solution of $\mathrm{AgNO}_{3}$ [18]. The color of the solution changed from greenish gray to colorless. $1 \mathrm{~g}$ of natural zeolite-Y was added to above mixture and stirred overnight. The product was collected and washed with distilled water and then dried at $100{ }^{\circ} \mathrm{C}$ for $2 \mathrm{~h}$. Finally, the AgNPs/Zeo-Y nanocomposite was collected for further studies.

\subsection{Synthesis of $\mathrm{AgCl} / \mathrm{Zeo}-\mathrm{Y}$}

The $\mathrm{Ag}^{+}$ion loaded Zeo-Y was placed in a sealed $100 \mathrm{ml}$ round bottom flask and then purged with $\mathrm{HCl}$ gas which was generated from $\mathrm{NaCl}$ in presence of conc. $\mathrm{H}_{2} \mathrm{SO}_{4}$ continuously to obtain $\mathrm{AgCl}$ immobilized Zeo-Y for a period of $2 \mathrm{~h}$.

\subsection{Preparation of AgNPs/Zeo-Y using lemon juice as reducing agent}

To $3 \mathrm{~mL}$ of DD water was added a fresh $1 \mathrm{~mL}$ of lemon juice and the $\mathrm{pH}$ of the medium was adjusted to 11 followed by $0.01 \mathrm{M}$ of silver nitrate solution was added. Finally, the yellowish green colored solution was changed to reddish brown color which indicated the formation of silver nanoparticle immobilized zeolites.

\subsection{Preparation of Amoxicillin modified AgNPs/Zeo-Y}

A known amount of AgNPs/Zeo-Y was dispersed an aqueous solution of 0.01M amoxicillin and then allowed stand for $1 \mathrm{~h}$ to obtain amoxicillin protected AgNPs/Zeo-Y. The final product was obtained by centrifugation.

\subsection{Instrumentation}

UV-Visible spectral studies were carried out using Shimadzu UV-Visible Spectrophotometer, Japan (Model UV-1800). The XRD patterns with diffraction intensity versus $2 \theta$ were recorded in a JSO Debye Flex 2002 Seifert diffractometer using $\mathrm{Cu} \mathrm{K} \alpha$ radiation $\left(\lambda=1.5406 \AA\right.$ ) from 10 to $80^{\circ}$ at a scanning speed of $1^{\circ} \min ^{-1}$. X-ray tube voltage and current were set at $40 \mathrm{kV}$ and $40 \mathrm{~mA}$, respectively. Morphological and structural investigations were carried out using field emission scanning electron microscopy (FE-SEM, SU6600, Hitachi, Japan).

\subsection{Antibacterial test}

To evaluate the antibacterial properties of AgNPs/Zeo-Y and amoxicillin modified AgNPs/Zeo-Y, Bacillus subtilis and Salmonella typhi were selected as Gram-negative and Gram-positive bacteria, respectively. One has the ability to measure the effectiveness of an antibacterial agent by determining a zone of inhibition. A standard inoculum of the test organism with $1 \times 10^{7}$ colony forming units (CFU)/mL was swabbed onto the surface of a LB agar plate, AgNPs/Zeo-Y and Amoxicillin/AgNPs/Zeo-Y antibacterial agents were placed on the surface of agar. The plates were incubated overnight at $37^{\circ} \mathrm{C}$, and the clear zones around the antibacterial agents were then measured. The above experiments were repeated thrice and the average values were taken.

\section{Results and discussion}

\subsection{Characterization of AgNPs loaded Zeolite Y}

The UV-Visible spectra of a) AgNPs/Zeo-Y, b) lemon juice, c) amoxicillin and d) AgNPs/Zeo-Y with amoxicillin are shown in Fig. 1. The silver nanoparticles loaded zeolite Y nanocomposite shows a silver nanoparticle peak at $420 \mathrm{~nm}$ due to surface plasmon resonance (SPR). The lemon juice not showing any peak and the amoxicillin peak was obtained at $270 \mathrm{~nm}$. After the incorporation of amoxicillin drug into the silver nanoparticles loaded zeolite Y nanocomposite two major peaks were observed one at $360 \mathrm{~nm}$ and another one at $250 \mathrm{~nm}$. Thus, these results confirm that amoxicillin drug was effectively attached to the silver nanoparticles loaded zeolite Y nanocomposite through ionic interaction.

The X-ray diffraction pattern of a) zeolite-Y and b) AgNps/Zeo-Y nanocomposite are shown in Fig. 2. As shown in Zeo-Y, the peaks at 31.04, 32.74, 33.24, 34.34, 35.94, 36.64, 37.76, 40.24, 41.74, 41.92, 52.80, 54.48, $56.54,57.58,58.74,65.22,66.54,69.34,71.10,72.92,75.96$, and $77.82^{\circ}$ are assigned for the presence of zeolite (JCPDS: 01-072-2344) [19]. In AgNPs/Zeo-Y, the characteristics peaks are due to the presence of silver chloride are attributed at 27.6, 32.05, 46.05, 54.6, 57.3, 67.4, 74.5 and $76.6^{\circ}$ with plane of $111,200,220,311,222,400$, 311 and 420 reflections owing to the $\mathrm{AgCl}$ phase. These results confirm that the silver nanoparticles loaded within zeolite Y matrix.

The structural and morphology were confirms by FE-SEM images. The mesoporous material of zeolite has octahedral shaped particles with various sizes around $1 \mu \mathrm{m}$ (Fig. 3(a)) [20]. From AgNPs loaded zeolite Y, the 


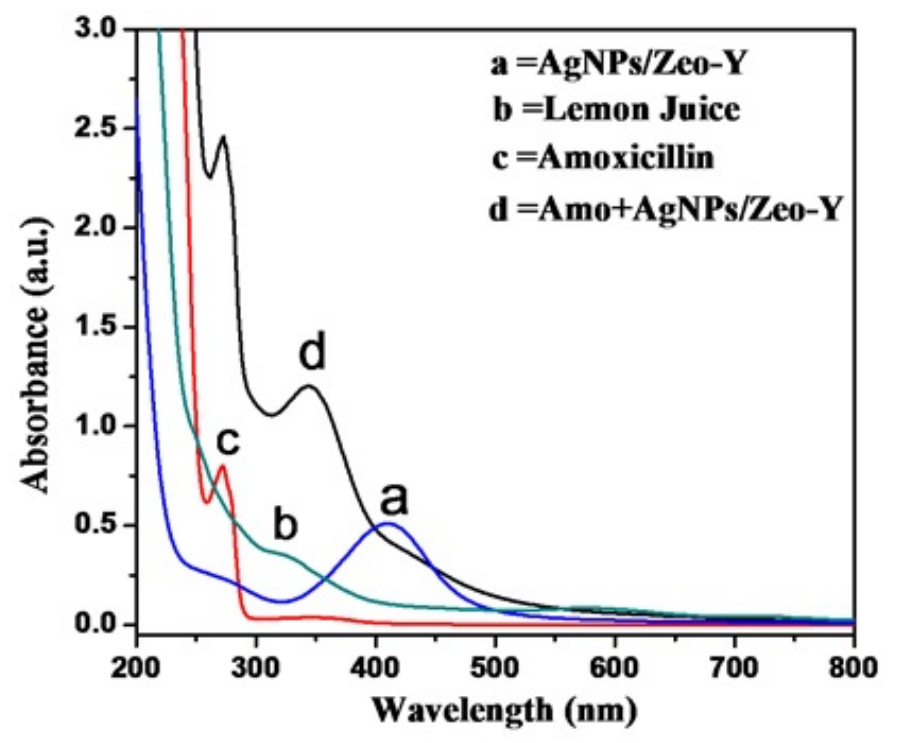

FIG. 1. UV-Visible spectrum of a) AgNPs/Zeo-Y, b) lemon, c) amoxicillin and d) AgNPs/Zeo-Y with amoxicillin

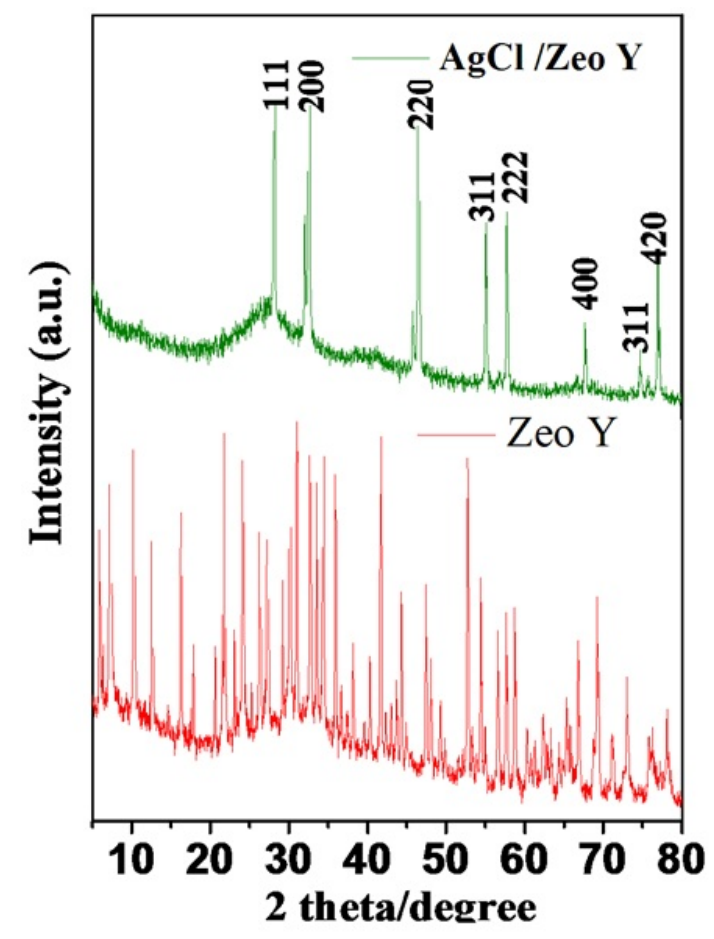

FIG. 2. XRD analysis a) zeolite $\mathrm{Y}$ and b) AgNPs loaded Zeo-Y 
silver nanoparticles loaded on surface of mesoporous zeolite and also regular shaped silver nanoparticles and the size of the silver nanoparticles are roughly $0.1 \mu \mathrm{m}$ as shown in Fig. 3(b). Therefore, the present method can be exploited for the effective loading of silver nanoparticles on zeolite surface.
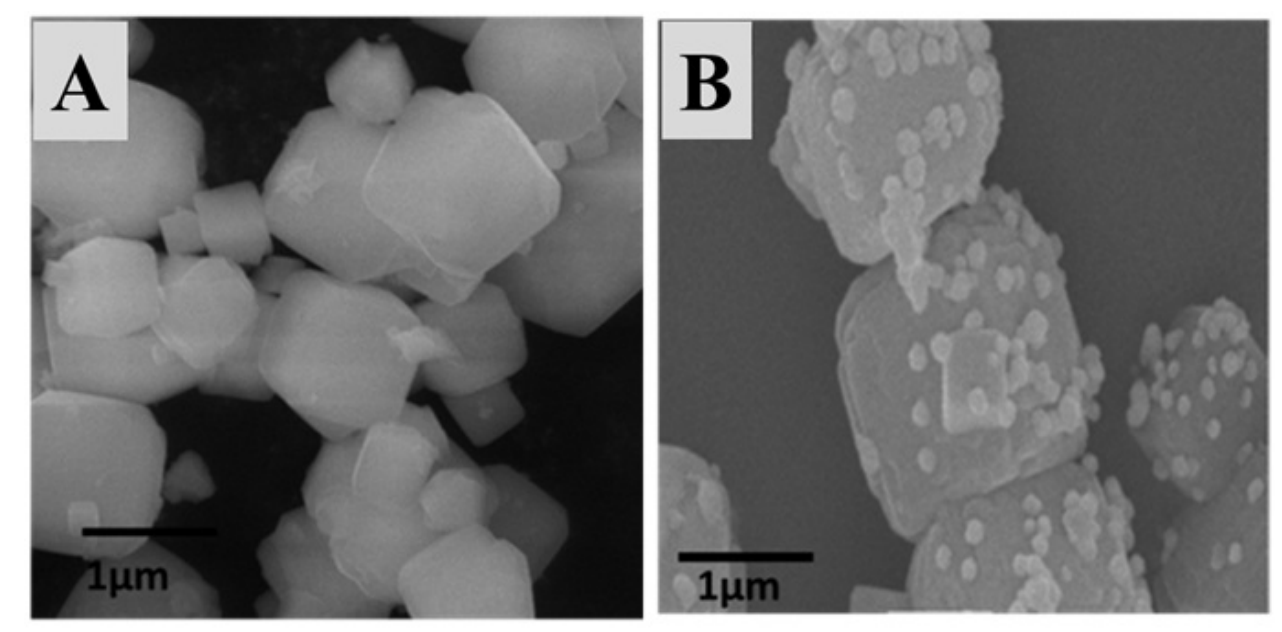

FIG. 3. FE-SEM images for Zeolite-Y (A) and AgNPs/Zeo-Y (B)

\subsection{Catalytic activity of Zeolite Y loaded AgNPs}

The catalytic activity of AgNPs/Zeo-Y was tested against the catalytic reduction of 4-Nitrophenol (4-NP) in the presence of $\mathrm{NaBH}_{4}$. Upon the addition of $\mathrm{NaBH}_{4}$ (Fig. 4(a)), the absorption peak of 4-Nitrophenol undergoes an immediate red-shift from $317 \mathrm{~nm}$ to $400 \mathrm{~nm}$ indicating the formation of 4-Nitrophenolate ions, evidenced by the visible color changes from light yellow to yellow green in color. After the addition of AgNPs/Zeo-Y using lemon juice nanocomposite, the absorption peak at $400 \mathrm{~nm}$ gradually decreased in intensity along with increase in absorption of a new peak at $300 \mathrm{~nm}$, indicating the formation of 4-Aminophenol (4-AP). Under these reaction conditions, the reduction was completed within 10 and 5 mins for the addition catalytic amount $5 \mathrm{mg}$ and $10 \mathrm{mg}$ respectively (Fig. 4(b) and Fig. 4(c)).

The rate of reaction forb the reduction of 4-NP in presence of the AgNPs/Zeo-Y nanocomposite is due to decrease in absorbance values at $400 \mathrm{~nm}$. Therefore, the rate constant of the reaction can be calculated using the following formula:

$$
\ln C / C_{0}=-k t
$$

where $k$ is the constant, $t$ is the reaction time; $C$ and $C_{0}$ are the concentration of 4-NP at time $t$ and 0 , respectively. The concentration or borohydride used in the entire study was $0.1 \mathrm{M}$, which is large excess when compared to the concentration of 4-NP, thus the reduction reaction is considered to be pseudo first order so that the rate of the reaction depends primarily on the concentration of 4-NP. The rate of the reaction $(k)$ obtained from the slope of the straight line was found to be $0.083 \mathrm{~min}^{-1}$ and $0.32 \mathrm{~min}^{-1}$ for 5 and $10 \mathrm{mg}$ of catalyst used for the catalytic studies (Fig. 4(d)). The rate of the reaction increases with increasing of the catalyst concentration which is due to the increase of the catalytic activities.

\subsection{Antibacterial activity}

It is well established that silver nanoparticles modified substrates have shown an enhanced antimicrobial activity $[12,13]$. In the present study, we studied the antimicrobial activity of silver nanoparticles, AgNPs/Zeo-Y and amoxicillin incorporated AgNPs/Zeo-Y against various Gram negative and Gram positive bacteria. Obviously, amoxicillin modified AgNPs/Zeo-Y showed a good antimicrobial activity against Bacillus subtilis and Salmonella typhi. The antimicrobial activity of AgNPs/Zeo-Y and Amoxicillin/AgNPs/Zeo-Y against various Gram positive and Gram negative bacteria as shown in Table 1.

\section{Conclusion}

In summary, the biogenic method was established to synthesize silver nanoparticle-loaded zeolite Y through an ion exchange approach followed green chemical reduction using lemon juice. This method is a fast and easy way to synthesize the AgNPs/Zeo-Y in large scale. The activity of AgNPs/Zeo-Y was studied, displaying excellent 

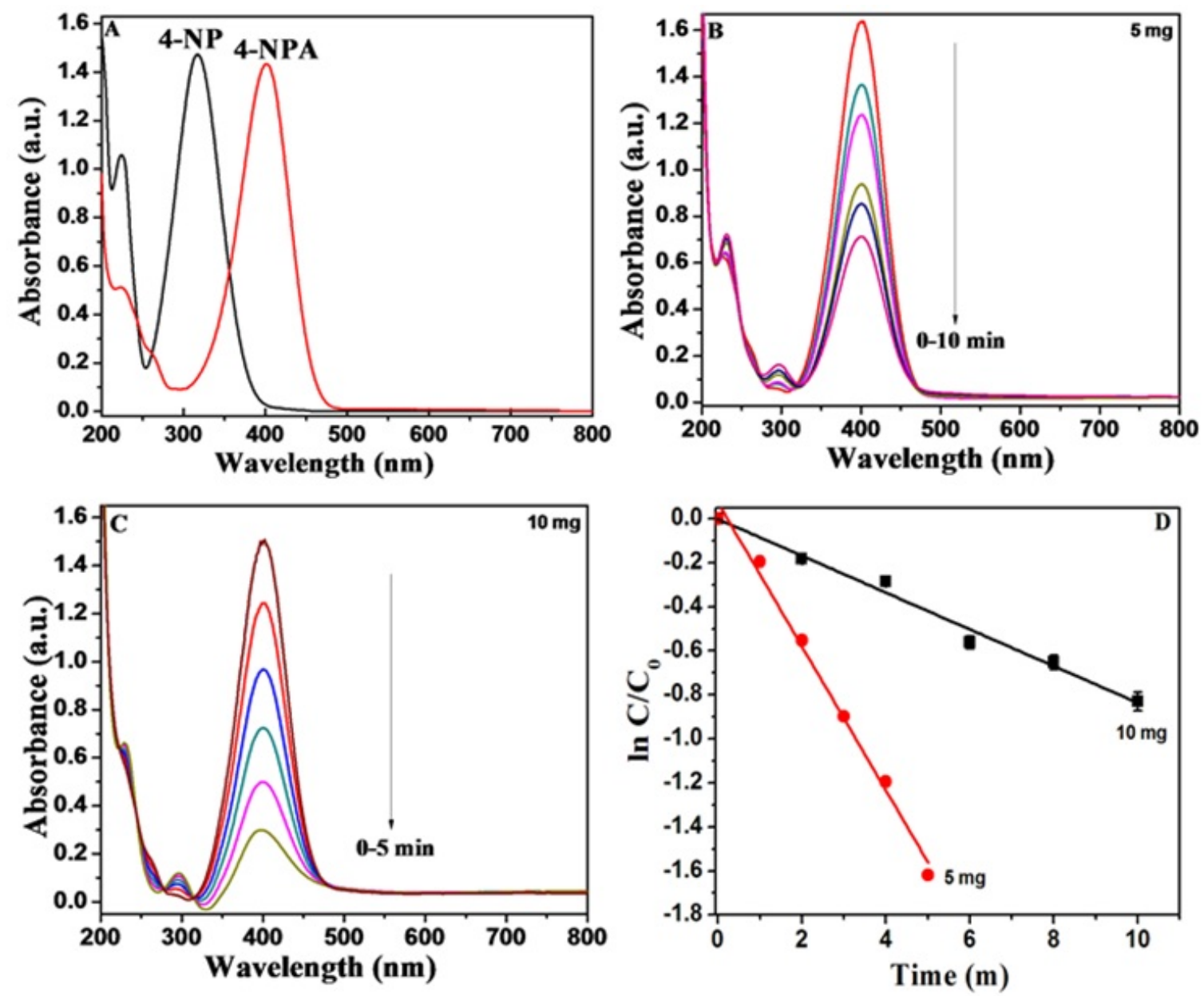

FIG. 4. UV-Visible spectra capturing the conversion of 4-NP to 4-AP upon reaction with $\mathrm{NaBH}_{4}(\mathrm{~A})$, in presence of $5 \mathrm{mg}$ and $10 \mathrm{mg}$ of AgNPs/Zeo-Y (B), (C) and kinetic plot. Error bars designates the standard deviation for five independent measurements (D)

TABLE 1. Antibacterial activity of AgNPs/Zeo-Y and Amoxicillin/AgNPs/Zeo-Y against Bacillus subtilis and Salmonella typhi

\begin{tabular}{|c|c|c|c|c|c|c|c|c|c|c|}
\hline \multirow{2}{*}{ Sample (mg/mL) } & \multicolumn{4}{|c|}{ Gram Positive Bacteria } & \multicolumn{4}{c|}{ Gram Negative Bacteria } \\
\cline { 2 - 12 } & \multicolumn{4}{|c|}{ Bacillus subtilis } & \multicolumn{5}{c|}{ Salmonella typhi } \\
\cline { 2 - 11 } & a & b & c & d & e & a & b & c & d & e \\
\hline AgNPs/Zeo-Y & 2.2 & 6.5 & 7.3 & 16.4 & - & 3.3 & 6.5 & 8.4 & 20.5 & - \\
\hline Amoxicillin AgNPs/Zeo-Y & - & 3.1 & 3.5 & 7.5 & - & - & - & - & 9.4 & - \\
\hline
\end{tabular}

$\mathrm{a}=(25 \mu \mathrm{g} / \mathrm{ml}), \mathrm{b}=(50 \mu \mathrm{g} / \mathrm{ml}), \mathrm{c}=(75 \mu \mathrm{g} / \mathrm{ml}), \mathrm{d}=(25 \mu \mathrm{g} / \mathrm{ml}$ (Standard) chloramphenicol), $\mathrm{e}=50 \mu \mathrm{g} / \mathrm{ml} 0.1 \%$ DMSO 
catalytic activity for the conversion of 4-NP to 4-AP. The amoxicillin-protected AgNPs/Zeo-Y system can be exhibited an enhanced antimicrobial activity as compared to AgNPs/Zeo-Y.

\section{Acknowledgement}

The authors (Dr. K.P. and S. M.) is grateful the UGC-CPEPA for providing partial financial assistance to carry out a part of this work.

\section{References}

[1] Bandura L., Franus M., Jozefaciuk G., Franus W. Synthetic zeolites from fly ash as effective mineral sorbents for land-based petroleum spills cleanup. Fuel, 2015, 147, P. 100-107.

[2] Rees L.V.C., Zuyi T. Rare-earth ion exchange in zeolite Y. Zeolites, 1986, 6, P. 201-205.

[3] Yu J., Xiao F., Corma A. Preface to special issue on zeolite materials and catalysis. Chinese Journals of Catalysis, 2015 , 36, P. 787-788.

[4] Serban S., Murr N.E. Synergetic effect for NADH oxidation of ferrocene and zeolite in modified carbon paste electrodes: New approach for dehydrogenase based biosensors. Biosensors and Bioelectronics, 2004, 20, P. 161-166.

[5] Onyango M.S., Kojima Y., Aoyi O., Bernardo E.C., Matsuda H. Adsorption equilibrium modeling and solution chemistry dependence of fluoride removal from water by trivalent-cation-exchanged zeolite F-9. Journal of Colloid and Interface Science, 2004, 279, P. 341-350.

[6] Banerjee R., Furukawa H., Britt D., Knobler C., Keeffe M.O., Yaghi O.M. Control of pore size and functionality I isoreticular zeolitic imidazolate frameworks and their carbon dioxide selective capture properties. J. Am. Chem. Soc., 2009, 131, P. 3875-3877.

[7] Zhang Y., Yu X., Wang X., Shan W., Yang P., Tang Y. Zeolite nanoparticles with immobilized metal ions:isolation and MALDI-TOFMS/MS identification of phosphopeptides. Chem. Commun., 2004, 25, P. 2882-2883.

[8] Mitsuo Y. Immobilization of toxic heavy metals using hydrotalcite minerals: possible applications for decontamination of polluted land and water. Journal of Ecotechnology Research, 2002, 8, P. 248-249.

[9] Kharlamov V.V., Minachev K.M., Gulyaeva L.P., Slyunadev P.I. The investigation of redox properties of palladium-zeolite catalysts. Russian Chemical Bulletin, 1995, 44, P. 621-623.

[10] Peter A., Cozmuta L.M., Cozmuta A.M., Nicula C. Photocatalytic efficiency of zeolite-based $\mathrm{TiO}_{2}$ composites for reduction of Cu (II): Kinetic models. Int. J. Appl. Ceram. Technol., 2014, 11, P. 568-581.

[11] Guzman M., Dille J., Godet S. Synthesis and antibacterial activity of silver nanoparticles against gram-positive and gram-negative bacteria. Nanomedicine: Nanotechnology, Biology and Medicine, 2012, 8, P. 37-45.

[12] Castanon G.A.M., Martinez N.N., Gutierrez F.M., Mendoza J.R., Ruiz F. Synthesis and antibacterial activity of silver nanoparticals with different sizes. J. Nanopart Res, 2008, 10, P. 1343-1348.

[13] Rai M., Yadav A., Gade A. Silver nanoparticals as a new generation of antimicrobials. Biotechn. Adv., 2009, 27, P. 76-83.

[14] Xie W.R.L.X.B., Shi Q.S., Zeng H.Y., Yang Y.S.O., Chen Y.B. Antibacterial activity and mechanism of silver nanoparticles on Escherichia coli. Appl. Microbial Biotechol, 2010, 85, P. 1115-1122.

[15] Baker C., Pradhan A., Pakstis L., Darrin J. Pochan, Shah S. Ismat. Synthesis and antibacterial properties of silver nanoparticles. J. Nanosci. Nanotech., 2005, 5, P. 244-249.

[16] Garza M.R., Olguin M.T., Sosa I.G., Alcantara D., Fuentes G.R. Silver supported on natural Mexican zeolite as an antibacterial material. Microporous and Mesoporous Materials, 2000, 39, P. 431-444.

[17] Kaur B., Srivastava R., Satpati B. Silver nanoparticle decorated polyaniline zeolite nanocompoite material based non-enzymatic electrochemical sensor for nanomolar detection of lindane. RSC Adv., 2015, 5, P. 57657-57665.

[18] Meenakshi S., Devi S., PAndian K., Devendiran R., Selvaraj M. Sunlight assisted synthesis of silver nanoparticles in zeolite matrix and study of its application on electrochemical detection of dopamine and uric acid in urine samples. Mat. Sci. Eng. C, 2016,69, P. 85-94.

[19] Shameli K., Ahmad M.B., Zargar M., Yunus W.M.Z.W., Ibrahim N.A. Fabrication of silver nanparticles doped in the zeolite framework and antibacterial activity. Int. J. Nanomed., 2011, 6, P. 331-341.

[20] Reddy V.R., Currao A., Calzaferri G., Zeolite A., Zeolite L. Monolayers modified with AgCl as photocatalyst for water oxidation to $\mathrm{O}_{2}$. J. Mater. Chem., 2007, 17, P. 3606-3609. 